\title{
Systemic immune response associated with radiation therapy in B-cell non-Hodgkin's lymphoma of Waldeyer's ring
}

\author{
XINGJIAN NIU ${ }^{1 *}$, HONGFEI JI ${ }^{2,3 *}$, YIRAN WANG ${ }^{2,3}$, SONGLIU HU $^{4}$, QIJIA XUAN ${ }^{1}$, LAN HUANG ${ }^{1}$, \\ LEI YIN ${ }^{2,3}$, WENJIA SU ${ }^{5}$, LIRU LI ${ }^{6}$, HAN ZHANG ${ }^{1}$, JINGTONG LI $^{1}$, YUE YANG ${ }^{2,3}$, \\ WEIWEI AN ${ }^{2,3}$ and QINGYUAN ZHANG ${ }^{1-3,6}$
}

${ }^{1}$ Department of Medical Oncology, Harbin Medical University Cancer Hospital; ${ }^{2}$ Institute of Cancer Prevention and Treatment;

${ }^{3}$ Heilongjiang Academy of Medical Sciences; ${ }^{4}$ Department of Radiation Oncology, Harbin Medical University Cancer Hospital;

${ }^{5}$ Department of Hematology, The First Affiliated Hospital of Harbin Medical University; ${ }^{6}$ Precision Medicine Center, Harbin Medical University Cancer Hospital, Harbin, Heilongjiang 150081, P.R. China

Received January 25, 2018; Accepted September 3, 2018

DOI: $10.3892 /$ or.2018.6748

\begin{abstract}
Radiation therapy (RT) is one of the most effective therapeutic modalities for B-cell non-Hodgkin's lymphoma of Waldeyer's ring (WR-B-NHL). However, the responsiveness of $\mathrm{RT}$ remains controversial and clinical biomarkers are required to predict survival in RT-treated patients with WR-B-NHL. Previous studies have suggested an association between RT and systemic immune responses. In the present retrospective study, the lymphocyte to monocyte ratio (LMR) was identified as a systemic immune indicator in RT-treated patients with WR-B-NHL, and the prognostic value of the LMR with RT and systemic immune responses were evaluated. The optimal cut-off value of the LMR was selected as 3.14, and a high LMR demonstrated improved prognosis and was considered an independent prognostic indicator in RT-treated patients, particularly in patients with distant non-irradiated lesions. Furthermore, reverse transcription-quantitative polymerase chain reaction and ELISA analysis of irradiated lymphoma cell lines and serum samples from patients with WR-B-NHL demonstrated the upregulated expression levels of 4-1BB ligands, calreticulin and high mobility group box 1 compared with non-irradiated groups. Additionally, $\mathrm{CD} 8^{+} \mathrm{T}$ cells and expression levels of interferon- $\gamma$ in $\mathrm{T}$ cells co-cultured with irradiated cells were significantly increased compared with non-irradiated cells. The results indicated that
\end{abstract}

Correspondence to: Dr Qingyuan Zhang, Department of Medical Oncology, Harbin Medical University Cancer Hospital, 150 Haping Road, Harbin, Heilongjiang 150081, P.R. China

E-mail: zqyHMU1965@163.com

*Contributed equally

Key words: B-cell non-Hodgkin's lymphoma of Waldeyer's ring, systemic immune response, radiation therapy, lymphocyte to monocyte ratio, prognosis the anti-programmed cell death protein 1 (PD-1) antibody may serve a role in lymphoma therapy when combined with RT. The results of the present study demonstrated the prognostic significance of the LMR associated with RT in patients with WR-B-NHL and acknowledged the potential use of PD-1 antibody in RT-treated lymphomas.

\section{Introduction}

Non-Hodgkin's lymphoma (NHL) comprises a clinically and pathologically heterogeneous group of disorders originating from lymphoid tissue, and the majority of all NHLs arise from B lymphocytes (B-NHL) (1). Waldeyer's ring is defined as a circular band of extranodal lymphoid tissues consisting of palatine tonsils, soft palate, nasopharynx, base of tongue, and oropharyngeal wall, which is the most common site of B-NHL in head and neck area (2-4). Due to the specific location of lesion, chemotherapy combination with radiation therapy (RT) is currently applied for the primary treatment in B-NHL of Waldeyer's ring (WR-B-NHL) (5,6). However, the effectiveness of RT is controversial and the clinical responses of patients with B-NHL treated with RT are heterogeneous $(7,8)$. Therefore, effective clinical prognostic biomarkers are required to predict the response of patients with WR-B-NHL to RT.

Increasing evidence has demonstrated an association between RT and immune system (9-12). The immune system has been reported to serve an important role in RT, whereby systemic immune responses are able to affect the therapeutic efficacy of radiation (9). Furthermore, RT has been suggested to serve as an immunogenic stimulus, which has the potential of inducing immunogenic cell death, and subsequently enhancing tumor-specific immunity within and outside the radiation field (13-16). When tumor cells are irradiated, they expose or release radiation-associated antigens for local and systemic disease regulation, including the activation of immune cells and trigger of specific anti-tumor responses $(11,17,18)$. Thus, immune system, including the innate and adaptive immune response, not only reflects the therapeutic effects of RT, but also provides a better understanding of RT-mediated systemic immune response. 
Clinically, surrogate markers based on systemic immunity, such as, peripheral blood parameters, including the absolute lymphocyte count (ALC), absolute monocyte count (AMC), lymphocyte to monocyte ratio (LMR), neutrophil to lymphocyte ratio (NLR), are considered immunologically relevant, and have been reported to aid in the prognostic evaluation of various types of malignancies (19-24). To date, it remains unclear which immune marker predicts clinical outcomes of $\mathrm{RT}$ and is associated with RT affecting systemic immunity in patients with WR-B-NHL. The present study aimed to investigate the prognostic value of systemic immune markers in patients with WR-B-NHL treated with RT, and confirmed the potential association between RT and systemic immune responses.

\section{Materials and methods}

Patients. The clinicopathological data of 164 patients who were diagnosed with WR-B-NHL between January 2008 and December 2012 at Harbin Medical University Cancer Hospital and The First Affiliated Hospital of Harbin Medical University (Harbin, China) were retrospectively reviewed in the present study. The male-to-female ratio was 1.38:1, and the median age for this cohort was 50 years old (range, 11-82 years old). Eligible participants were pathologically confirmed with B-NHL according to the World Health Organization classification of Tumors of Haematopoietic and Lymphoid Tissues (25), with no previous history of malignancy, immunosuppression or transplantation. The clinicopathological characteristics of the patients, including sex, age, Ann Arbor stage, distant lesion involvement, Eastern Cooperative Oncology Group performance status (ECOG PS), systemic B symptoms, international prognostic index (IPI) score, and serum lactate dehydrogenase (LDH) level are listed in Table I. All patients received standard combination CHOP (cyclophosphamide, doxorubicin, vincristine, and prednisone) or rituximab (R)-CHOP chemotherapy regimens followed by $\mathrm{RT}$. RT was given according to the involved nodal field or organ, as previously described (26). The median dose was 36 Gy in 1.8-2 Gy fractions, given 3-6 weeks after chemotherapy. The study protocol was approved by the Institutional Review Board of Harbin Medical University Ethics Committee. Written informed consent was obtained from all participants, whereby informed consent from patients $<16$ years old was obtained from their parents or guardians. All methods were performed in accordance with the relevant guidelines and regulations.

Blood sample analysis. Peripheral blood samples were obtained from enrolled patients within 1 week before RT in the retrospective study. Data on standard automated complete blood counts (CBC) of blood samples were counted using Sysmex XT-1800 Automated Hematology system (Sysmex Corporation, Kobe, Japan). ALC and AMC levels were derived from CBC tests. The peripheral LMR was calculated as the ratio of the ALC to AMC. The peripheral NLR was shown as the ratio between of absolute neutrophil counts (ANC) and ALC.

Cell lines. In the current study, the human diffuse large B cell line, SU-DHL-4, and human Burkitt lymphoma cell line, Raji, were obtained from the American Type Culture
Collection (ATCC nos. CRL-2957 and CCL-86, respectively; Manassas, VA, USA). The cells were cultured in RPMI-1640 supplemented with $10 \%$ fetal bovine serum (FBS) (both from Gibco; Thermo Fisher Scientific, Inc., Waltham, MA, USA) and $1 \%$ penicillin-streptomycin in a humidified atmosphere with $5 \% \mathrm{CO}_{2}$ at $37^{\circ} \mathrm{C}$.

Cell irradiation. SU-DHL-4 and Raji cells were treated with 1, 3 and 5 Gy of 4MV X-ray irradiation generated by a high-energy linear accelerator (Elekta Synergy, Stockholm, Sweden) at a dose rate of $2 \mathrm{~Gy} / \mathrm{min}$ at room temperature. Following treatment, the cells were incubated at $37^{\circ} \mathrm{C}$ for $24 \mathrm{~h}$, and then were counted using an automatic cell counter. Subsequently, cells were centrifuged at $400 \mathrm{x}$ g for $5 \mathrm{~min}$ at room temperature and then collected for further analysis.

Induction of lymphoma-reactive T-cell lines. Peripheral blood mononuclear cells (PBMCs) were isolated from healthy volunteers by density-gradient centrifugation using Ficoll-Hypaque (GE Healthcare, Chicago, IL, USA) according to the manufacturer's protocol. The present study was approved by the Harbin Medical University Cancer Hospital Ethics Committee and written informed consent was obtained from all healthy volunteers.

In order to obtain the lymphoma-reactive $\mathrm{T}$ cells, lymphoma tumor cells were induced with $800 \mathrm{ng} / \mathrm{ml}$ soluble CD40 ligand trimer (Amgen, Thousand Oaks, CA, USA) and then with $2 \mathrm{ng} / \mathrm{ml}$ IL-4 (PeproTech, Inc., Rocky Hill, NJ, USA) as previously reported (27). Subsequently, T cells were stimulated with the CD40L-activated tumor cells four times. Then, the lymphoma-reactive $\mathrm{T}$ cells were cultured in the presence of $10 \mathrm{IU} / \mathrm{ml}$ IL-2 (Proleukin, Chiron, Amsterdam, The Netherlands) for further assays.

Cell co-culture and treatment. Ten thousand non-irradiated or irradiated SU-DHL-4 and Raji cells were cultured in a 6-well culture plate in RPMI-1640 medium supplemented with $10 \%$ FBS. The T cells were added to the wells and co-cultured with non-irradiated or irradiated SU-DHL-4 and Raji cells at $37^{\circ} \mathrm{C}$ incubator with $5 \% \mathrm{CO}_{2}$. After $48 \mathrm{~h}$ of incubation, cells and culture media were collected and stored for further analysis.

Separate sets of experiments were performed for anti-programmed cell death protein 1 (PD-1) treatment to the co-culture systems. After cells were under co-culture systems for $24 \mathrm{~h}$, the anti-PD-1 antibody $(1 \mu \mathrm{g} / \mathrm{ml}$; R\&D Systems, Inc., Minneapolis, MN, USA) was added into the co-culture T and non-irradiated or irradiated lymphoma cells. Then, the cells were centrifuged at $400 \mathrm{x}$ g for $5 \mathrm{~min}$ at room temperature and the cell supernatant was collected.

RNA extraction and reverse transcription-quantitative polymerase chain reaction $(R T-q P C R)$ analysis. Total RNA was extracted from irradiated SU-DHL-4 and Raji cells using TRIzol reagent (Invitrogen; Thermo Fisher Scientific, Inc.), following the manufacturer's protocol. For cDNA synthesis, $2 \mu \mathrm{g}$ of total RNA was reverse transcribed using the Transcriptor First Strand cDNA Synthesis kit (Roche Diagnostics, Basel, Switzerland). qPCR analysis was performed using SYBR Premix Ex Taq II (Takara Bio, Inc., Otsu, Japan). The primers used are listed in Table II. The 
Table I. Clinicopathological characteristics of patients with WR-B-NHL based on LMR.

\begin{tabular}{|c|c|c|c|c|}
\hline \multirow[b]{2}{*}{ Characteristics } & \multirow[b]{2}{*}{ Overall $(\%)(n=164)$} & \multicolumn{3}{|c|}{ LMR } \\
\hline & & $\leq 3.14(\mathrm{n}=86)$ & $>3.14(\mathrm{n}=78)$ & P-value \\
\hline Sex & & & & $0.708^{\mathrm{a}}$ \\
\hline Male & $95(57.93)$ & 51 & 44 & \\
\hline Female & $69(42.07)$ & 35 & 34 & \\
\hline Age, years & & & & $0.791^{\mathrm{a}}$ \\
\hline$\leq 60$ & $114(69.51)$ & 59 & 55 & \\
\hline$>60$ & $50(30.49)$ & 27 & 23 & \\
\hline Histological subtype & & & & $0.002^{\mathrm{a}}$ \\
\hline DLBCL & $97(59.15)$ & 41 & 56 & \\
\hline Non-DLBCL & $67(40.85)$ & 45 & 22 & \\
\hline Ann Arbor stage & & & & $0.004^{\mathrm{a}}$ \\
\hline $\mathrm{I} / \mathrm{II}$ & $127(77.44)$ & 59 & 68 & \\
\hline III/IV & $37(22.56)$ & 27 & 10 & \\
\hline Distant lesion involvement & & & & $0.045^{\mathrm{a}}$ \\
\hline- & $107(65.24)$ & 50 & 57 & \\
\hline+ & $57(34.76)$ & 36 & 21 & \\
\hline ECOG PS & & & & $0.072^{\mathrm{a}}$ \\
\hline $0-1$ & $154(93.90)$ & 78 & 76 & \\
\hline$\geq 2$ & $10(6.10)$ & 8 & 2 & \\
\hline B symptoms & & & & $0.007^{\mathrm{a}}$ \\
\hline- & $123(75.00)$ & 57 & 66 & \\
\hline+ & $41(25.00)$ & 29 & 12 & \\
\hline IPI score & & & & $0.006^{\mathrm{a}}$ \\
\hline 0 & $64(39.02)$ & 28 & 36 & \\
\hline 1 & $47(28.66)$ & 20 & 27 & \\
\hline 2 & $34(20.73)$ & 23 & 11 & \\
\hline$\geq 3$ & $19(11.59)$ & 15 & 4 & \\
\hline LDH & & & & $0.623^{\mathrm{a}}$ \\
\hline Normal & $85(51.83)$ & 43 & 42 & \\
\hline High & $79(48.17)$ & 43 & 36 & \\
\hline Treatment regimens & & & & $0.888^{\mathrm{a}}$ \\
\hline CHOP plus RT & $119(72.56)$ & 62 & 57 & \\
\hline R-CHOP plus RT & $45(27.44)$ & 24 & 21 & \\
\hline $\operatorname{ALC}\left(x 10^{9} / 1\right)$ & $1.65(0.11-11.4)^{\mathrm{c}}$ & $1.22(0.11-2.44)^{\mathrm{c}}$ & $2.10(0.8-11.4)^{\mathrm{c}}$ & $0.335^{\mathrm{b}}$ \\
\hline $\operatorname{AMC}\left(\times 10^{9} / 1\right)$ & $0.52(0.08-2.55)^{\mathrm{c}}$ & $0.64(0.18-2.55)^{\mathrm{c}}$ & $0.39(0.08-1.9)^{\mathrm{c}}$ & $\leq 0.001^{\mathrm{b}}$ \\
\hline
\end{tabular}

thermocycling conditions were as follows: Initial step of $95^{\circ} \mathrm{C}$ for $2 \mathrm{~min}$; followed by 50 cycles of $95^{\circ} \mathrm{C}$ for $5 \mathrm{sec}, 58^{\circ} \mathrm{C}$ for $10 \mathrm{sec}$; and extension at $72^{\circ} \mathrm{C}$ for $30 \mathrm{sec}$. The relative quantification of mRNA expression was calculated by the $2^{-\Delta \Delta C q}$ method (28) following normalization to $\beta$-actin expression.

ELISA. The culture supernatants from irradiated SU-DHL-4 and Raji cells, and peripheral blood samples collected as aforementioned were used to measure the expression levels of 4-1BB ligands (4-1BBL), calreticulin (CRT) and high mobility group box 1 (HMGB1) via ELISA analysis. CRT levels were determined using double-antibody sandwich ELISA (cat. no. xl-Em1860, Shanghai Xin Le Biological Technology Co., Ltd., Shanghai, China). HMGB1 levels were measured using a commercial ELISA kit from Novatein Biosciences, Inc. (cat. no. NB-S11133; Woburn, MA, USA) 
Table II. Primer sequences for RT-qPCR.

\begin{tabular}{ll}
\hline Primer & \multicolumn{1}{c}{ Sequence (5'-3') } \\
\hline Calreticulin & \\
F & AAATGAGAAGAGCCCCGTTCTTCCT \\
R & AAGCCACAGGCCTGAGATTTCATCTG \\
HMGB1 & \\
F & TTGATTCTAATAATCCCATGCTTTGA \\
R & AATTTCACATAGCCCACTTACATTTAC \\
MICA/B & \\
F & GTGCCCCAGTCCTCCAGAGCTCAG \\
R & GTGGCATCCCTGTGGTCACTCGTC \\
FAS & \\
F & ATTATCGTCCAAAAGTGTTAAT \\
R & TGCATGTTTTCTGTACTTCCTT \\
OX-40 ligands & \\
F & TCACCTACATCTGCCTGCACTT \\
R & GAAACCTTTCTCCTTCTTATATTCGGTA \\
4-1BB ligands & \\
F & GTTTCACTTGCGCTGCACCTGCAGCCA \\
R & CTG \\
& GGCTCTAGATATCAAGGTCCAACTTGG \\
h-ACTB & GGAAGG \\
F & GGGAAATCGTGCGTGACATT \\
R & GGAACCGCTCATTGCCAAT \\
\hline
\end{tabular}

RT-qPCR, reverse transcription-quantitative polymerase chain reaction; F, forward; R, reverse; HMGB1, high mobility group box 1 .

and 4-1BBL levels were measured by an ELISA kit from RayBiotech, Inc. (cat. no. ELH-41BB; Norcross, GA, USA) according to the manufacturer's protocols. IFN- $\gamma$ production from the co-systems of T cells with non-irradiated or irradiated lymphoma cells were measured using an IFN- $\gamma$ ELISA kit from R\&D Systems, Inc. (cat. no. DIF50).

Flow cytometry. T cells co-cultured with non-irradiated or irradiated SU-DHL-4 cells were used for flow cytometry. The cells were stained with human-CD8-fluorescein isothiocyanate antibody (monoclonal RPA-T8; cat. no. 11-0088-42; 1:20; eBioscience; Thermo Fisher Scientific, Inc.) at room temperature for $20 \mathrm{~min}$. Following washing with staining buffer (cat. no. 00-4222-57; eBioscience; Thermo Fisher Scientific, Inc.), the stained cells were detected using a BD Biosciences LSR Fortessa and analyzed using BD Accuri C6 software (both from BD Biosciences, Franklin Lakes, NJ, USA).

Statistical analysis. The receiver operating characteristic (ROC) curve analysis was performed to select cut-off value of the ALC, AMC, LMR and NLR for survival analysis. The optimal cut-off point on the ROC curve was considered as the highest Youden index (sensitivity + specificity-1). Chi-squared and Fisher's exact tests were performed to evaluate the associations between the LMR and clinicopathological parameters.

The Kaplan-Meier estimator method was used to determine overall survival (OS; the period between the date of diagnosis and the date of mortality due to any cause or the last follow-up) and progression-free survival (PFS; the period between the date of RT initiation and the date of disease progression). Differences between OS or PFS curves were compared using the two-tailed log-rank test. Multivariate analyses to evaluate the variables under the prognostic factors section were performed using Cox proportional hazards models, and the results were presented as hazard ratios (HRs) and $95 \%$ confidence intervals (CIs). Two-tailed, unpaired Student's t-tests, one-way analysis of variance with Fisher's Least Significant Difference post hoc and Wilcoxon signed-rank test were used to analyze the statistical significance of RT-PCR, ELISA and flow cytometry results. Data are presented as the mean \pm standard deviation. All statistical data analyses were performed with SPSS 20.0 statistics software (IBM Corp., Armonk, NY, USA). $\mathrm{P}<0.05$ was considered to indicate a statistically significant difference.

\section{Results}

Clinical characteristics in RT-treated patients with $W R-B-N H L$. A total of 164 patients with WR-B-NHL who received RT were enrolled in the present study and the clinicopathological characteristics of these patients are listed in Table I. In a cohort of 164 patients, 97 patients $(59.15 \%)$ were diagnosed with diffuse large B-cell lymphoma and 67 patients (40.85\%) were diagnosed with other subtypes. According to the Ann Arbor stage, 127 patients (77.44\%) were in early stage (stage I and II) and 37 patients (22.56\%) were in the advanced stage (stage III and IV). Fifty-seven patients (34.76\%) had single or multiple distant lesions. According to the ECOG PS classification, 154 patients $(93.90 \%)$ were classified as grade $0-1$ and 10 patients $(6.10 \%)$ were classified as grade $\geq 2$. Additionally, 41 patients $(25.00 \%)$ had B symptoms and 53 patients (32.32\%) had intermediate or high-risk IPI scores. Based on serum LDH levels, 79 patients (48.17\%) had higher LDH levels over the upper limit of the normal range (normal range, $120-246 \mathrm{U} / \mathrm{l})$. All patients received CHOP (72.56\% of patients) or R-CHOP (27.44\% of patients) chemotherapy regimens combined with the RT.

LMR is a systemic immune marker to divide RT-treated patients with WR-B-NHL. The peripheral ALC, AMC, LMR and NLR were collected and calculated from patients with WR-B-NHL treated with RT to define the systemic immune status of these patients. Then, the four parameters were analyzed using the ROC curve (1). No statistically significant differences in the ALC, AMC or NLR were identified by ROC analyses (Fig. 1A, B and D). The significant optimal cut-off value of the LMR was selected as 3.14, with an area under the curve (AUC) value of 0.675 ( $\mathrm{P}=0.011$; Fig. 1C). The enrolled patients were classified into the low LMR group $(n=86)$ and high LMR group $(n=78)$ according to the cut-off LMR value, as presented in Table I.

The association between the LMR and clinical characteristics are stated in Table I. Patients with an LMR 

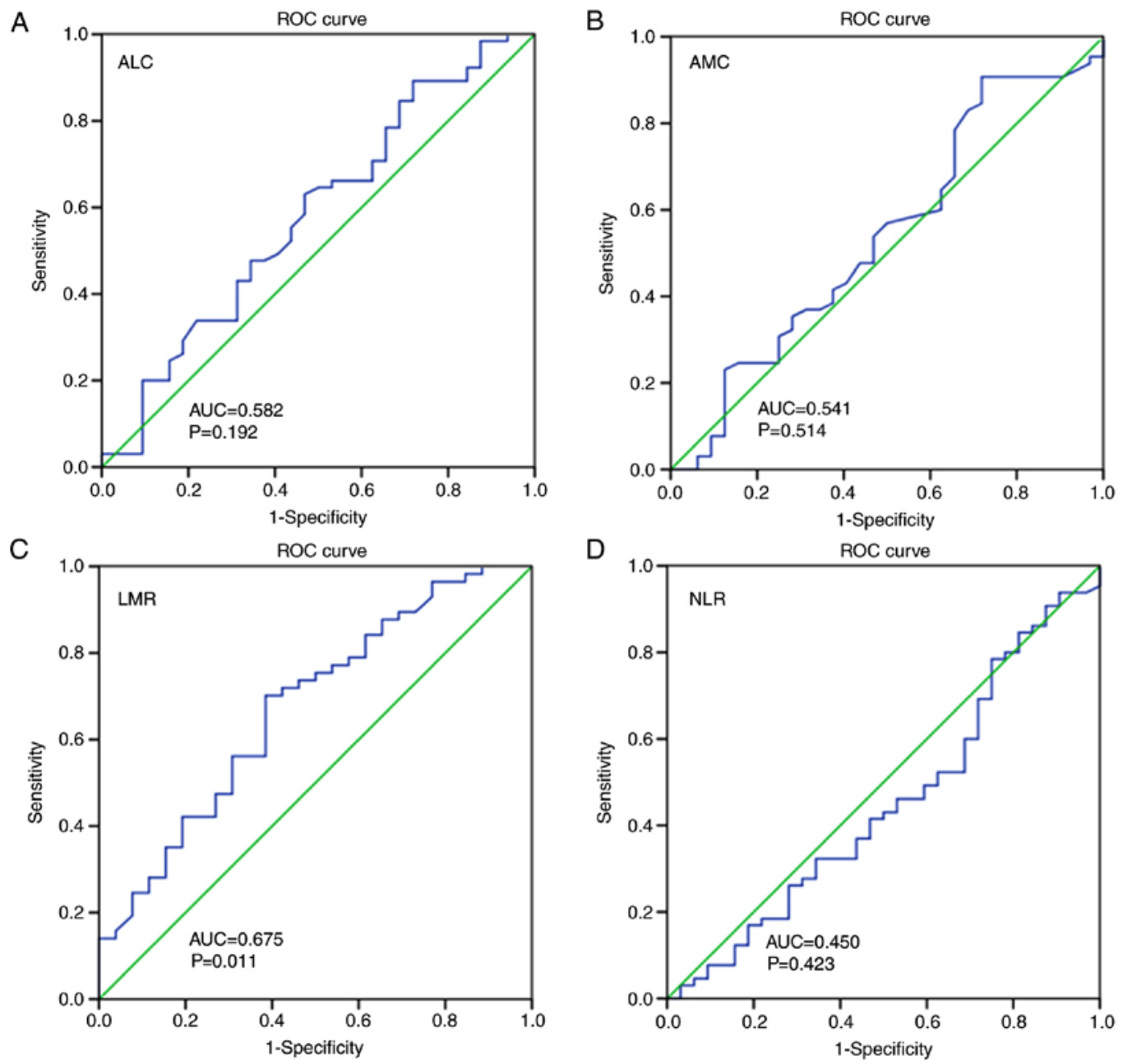

Figure 1. ROC curve analysis for several peripheral blood parameters in 164 WR-B-NHL patients. (A) ROC analysis for ALC (P=0.192 with an AUC as 0.582). (B) ROC analysis for AMC ( $\mathrm{P}=0.514$ with an AUC as 0.541). (C) ROC analysis for the LMR ( $\mathrm{P}=0.011$ with an AUC as 0.675 , the cut-off value was 3.14). (D) ROC analysis for the NLR ( $\mathrm{P}=0.423$ with an AUC as 0.450). ROC, receiver operating characteristic; WR-B-NHL, B cell non-Hodgkin's lymphoma of Waldeyer's ring; ALC, absolute lymphocyte count; AUC, area under the curve; AMC, absolute monocyte count; LMR, lymphocyte to monocyte ratio; NLR, neutrophil to lymphocyte ratio.
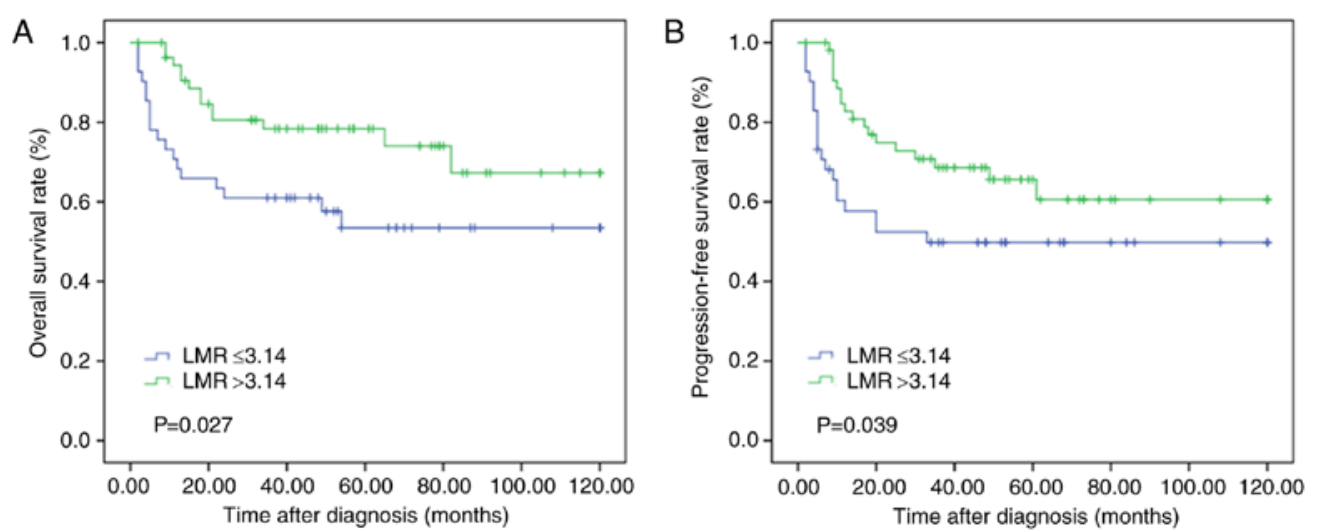

Figure 2. Kaplan-Meier estimated the OS and PFS in 164 WR-B-NHL patients treated with RT. The patients were divided into two groups according to the LMR cut-off value of 3.14. Patients with an LMR $>3.14$ presented significantly higher (A) OS rates ( $\mathrm{P}=0.027)$ and (B) $\mathrm{PFS}$ rates $(\mathrm{P}=0.039)$ compared with an LMR $\leq 3.14$. OS, overall survival; PFS, progression-free survival; WR-B-NHL, B cell non-Hodgkin's lymphoma of Waldeyer's ring; RT, radiation therapy; LMR, lymphocyte to monocyte ratio.

$\leq 3.14$ were significantly associated with the histological subtype $(\mathrm{P}=0.002)$, the advanced Ann Arbor stage $(\mathrm{P}=0.004)$ and IPI score $(\mathrm{P}=0.006)$, higher presence of $\mathrm{B}$ systems $(\mathrm{P}=0.007)$ and distant lesion involvements $(\mathrm{P}=0.045)$ (Table I). The mean counts of lymphocytes in the low or high LMR groups were $1.22 \times 10^{9} / 1$ (range, $\left.0.11 \times 10^{9}-2.44 \times 10^{9} / 1\right)$ and $2.10 \times 10^{9} / 1$ (range, 

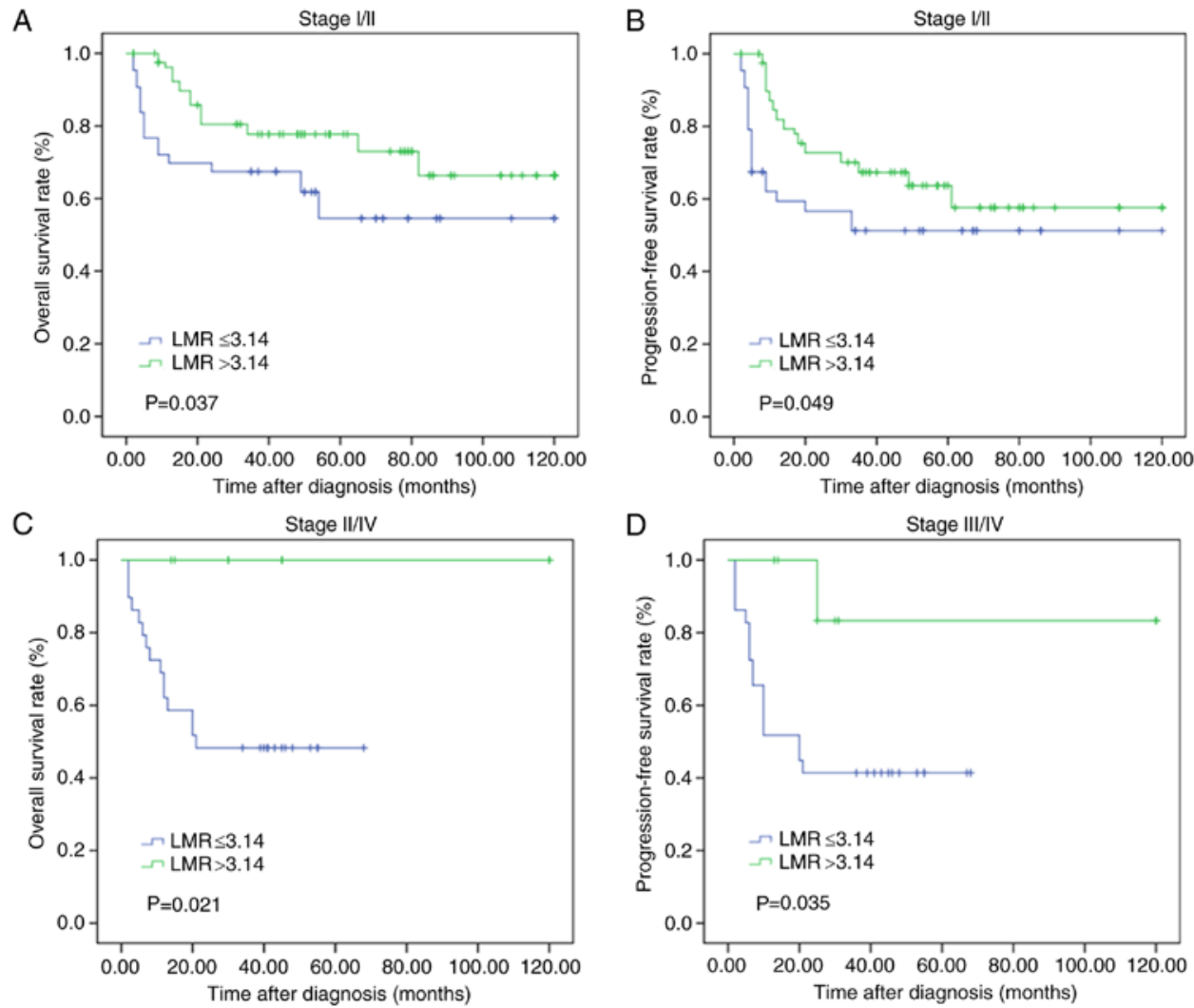

Figure 3. Kaplan-Meier estimated the OS and PFS rates in RT-treated WR-B-NHL patients with different clinical stages. In early stage (stage I/II), patients with an LMR $>3.14$ showed higher (A) OS rates $(\mathrm{P}=0.037)$ and (B) PFS rates $(\mathrm{P}=0.049)$ compared with an LMR $\leq 3.14$. In advanced stage (stage III/IV), patients with an LMR $>3.14$ showed higher $(C)$ OS rates $(P=0.021)$ and (D) PFS rates $(P=0.035)$ compared with an LMR $\leq 3.14$. OS, overall survival; PFS, progression-free survival; WR-B-NHL, B cell non-Hodgkin's lymphoma of Waldeyer's ring; RT, radiation therapy; LMR, lymphocyte to monocyte ratio.
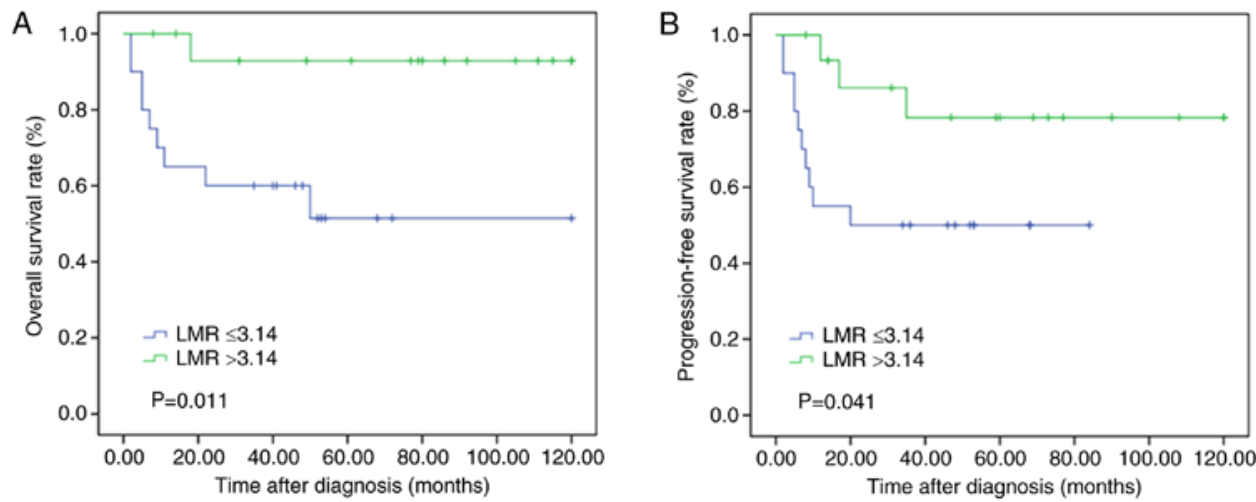

Figure 4. Kaplan-Meier estimated the OS and PFS rates in 57 RT-treated patients who had distant lesion involvements. Patients with an LMR $>3.14$ presented significantly higher (A) OS rates ( $\mathrm{P}=0.011)$ and (B) PFS rates $(\mathrm{P}=0.041)$ compared with an LMR $\leq 3.14$. OS, overall survival; PFS, progression-free survival; $\mathrm{RT}$, radiation therapy; LMR, lymphocyte to monocyte ratio.

$\left.0.8 \times 10^{9}-11.4 \times 10^{9} / 1\right)$, respectively. The mean counts of monocytes were $0.64 \times 10^{9} / 1$ (range, $0.18 \times 10^{9}-2.55 \times 10^{9} / 1$ ) in the low LMR group and $0.39 \times 10^{9} / 1$ (range, $0.08 \times 10^{9}-1.9 \times 10^{9} / 1$ ) in the high LMR group $(\mathrm{P}<0.001$; Table I).

A high LMR predicts improved prognosis in patients with $W R-B-N H L$ with RT. Kaplan-Meier curve analysis was performed to evaluate the OS and PFS rates in the low and high LMR groups. The OS and PFS rates were significantly worse in the LMR $\leq 3.14$ group compared with that in the LMR $>3.14$ group $(\mathrm{P}=0.027$ and $\mathrm{P}=0.039$, respectively; Fig. 2$)$.
LMR predicts survival in RT-treated patients with WR-B-NHL with clinical stages as well as in patients with distant lesion involvements. To further evaluate the effects of the LMR on patient survival with different subgroups of clinical stages, the enrolled patients were separated into low and high LMR groups according to early (stage I/II) and advanced (stage III/IV) stages. Using the Kaplan-Meier method, high LMR levels in patients with early and advanced stages were demonstrated to predict relatively longer $\mathrm{OS}(\mathrm{P}=0.037$ and $\mathrm{P}=0.049$, respectively) and PFS times $(\mathrm{P}=0.021$ and $\mathrm{P}=0.035$, respectively) (Fig. 3). 
Table III. Multivariate analysis of prognostic factors for survival in patients with WR-B-NHL.

\begin{tabular}{|c|c|c|c|c|c|c|}
\hline \multirow[b]{2}{*}{ Covariate } & \multicolumn{3}{|c|}{ OS } & \multicolumn{3}{|c|}{ PFS } \\
\hline & HR & $95 \% \mathrm{CI}$ & P-value ${ }^{a}$ & HR & $95 \% \mathrm{CI}$ & P-value ${ }^{a}$ \\
\hline Age, years & 0.825 & $0.373-1.824$ & 0.635 & 0.731 & $0.352-1.516$ & 0.399 \\
\hline Histological subtype & 1.297 & $0.513-3.280$ & 0.582 & 0.817 & $0.362-1.844$ & 0.627 \\
\hline Ann Arbor stage & 1.328 & $0.572-3.081$ & 0.509 & 1.458 & $0.654-3.248$ & 0.356 \\
\hline Distant lesion involvement & 0.597 & $0.229-1.554$ & 0.291 & 0.680 & $0.298-1.552$ & 0.360 \\
\hline ECOG PS & 1.305 & $0.338-5.048$ & 0.699 & 2.122 & $0.718-6.271$ & 0.173 \\
\hline B symptoms & 1.488 & $0.522-4.241$ & 0.457 & 1.097 & $0.421-2.862$ & 0.850 \\
\hline IPI score & 0.760 & $0.341-1.698$ & 0.504 & 0.612 & $0.289-1.293$ & 0.198 \\
\hline LDH & 0.602 & $0.267-1.356$ & 0.220 & 0.723 & $0.349-1.501$ & 0.385 \\
\hline Treatment regimens & 0.619 & $0.249-1.540$ & 0.302 & 0.496 & $0.211-1.164$ & 0.107 \\
\hline LMR & 0.427 & $0.197-0.926$ & 0.031 & 0.435 & $0.214-0.885$ & 0.022 \\
\hline
\end{tabular}

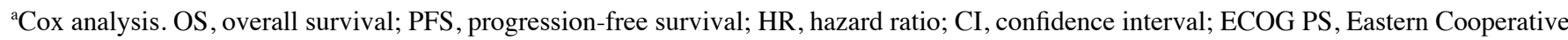
Oncology Group performance status; IPI, international prognostic index; LDH, lactate dehydrogenase; LMR, lymphocyte-to-monocyte ratio.
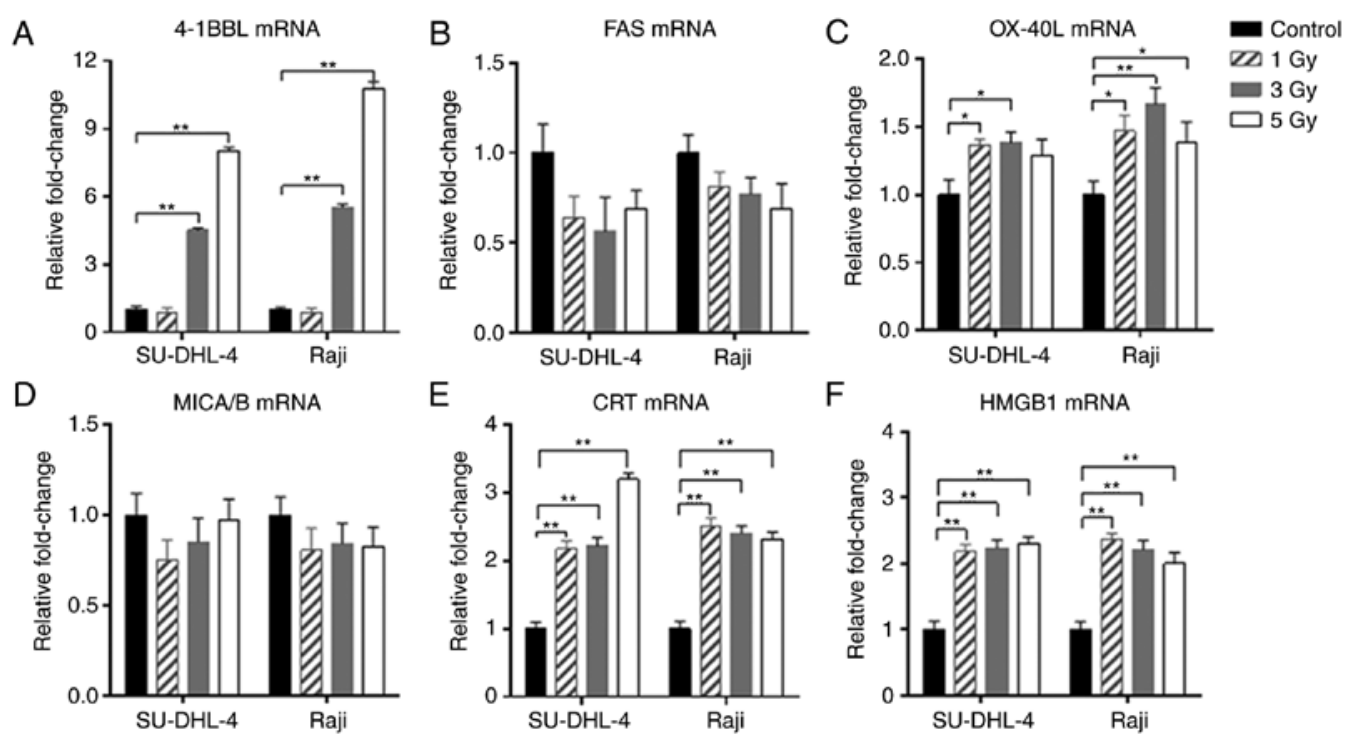

Figure 5. RT-PCR analysis of radiation-associated proteins in SU-DHL-4 and Raji cells treated with 0, 1, 3 and 5 Gy of 4MV X-ray irradiation. The radiation-associated proteins included: (A) 4-1BB ligands, (B) CRT, (C) HMGB1, (D) MICA/B, (E) FAS and (F) OX-40 ligands. "P<0.05 vs. Control; ** P $<0.01$ vs. Control. RT-PCR, reverse transcription-polymerase chain reaction; CRT, calreticulin; HMGB1, high mobility group box 1.

In a cohort of 164 patients, 57 patients received RT following the presence of simple or multiple distant lesions. The OS and PFS rates of the prognosis were evaluated in these patients. The curves revealed that patients who had higher LMR values exhibited significantly more improved clinical responses to RT compared with their matched counterparts with lower LMR values ( $\mathrm{P}=0.011$ and $\mathrm{P}=0.041$, respectively; Fig. 4).

LMR is an independent prognostic indicator in RT-treated patients with $W R-B-N H L$. The influences of various clinical characteristics on OS or PFS were estimated using Cox proportional hazards models in all 164 patients, as shown in Table III. According to the multivariate analyses, the LMR may be regarded as an independent prognostic indicator for OS ( $\mathrm{P}=0.031$; HR, 0.427; 95\% CI, 0.197-0.926) and PFS $(\mathrm{P}=0.022$; HR, 0.435; 95\% CI, 0.214-0.885). Other clinical variables were not significantly associated with OS or PFS in the multivariate analysis.

Overexpression of radiation-associated antigens occurs in irradiated lymphoma cells and serum samples of patient. SU-DHL-4 and Raji cells were irradiated to simulate RT in patients with B-NHL. A total of six potential radiationassociated proteins were selected and analyzed as they are considered as important radiation-induced proteins associated with $\mathrm{T}$ cell responses (29). The results demonstrated that following radiation, the mRNA expression of 4-1BBL, CRT, HMGB1 and OX-40 ligands (OX-40L) were higher compared with non-irradiated cells, whereas NKG2D ligands (named MICA/B) and FAS were unchanged compared with the control group (Fig. 5). Additionally, the soluble expressions of 4-1BBL, CRT and HMGB1, which were notably upregulated 

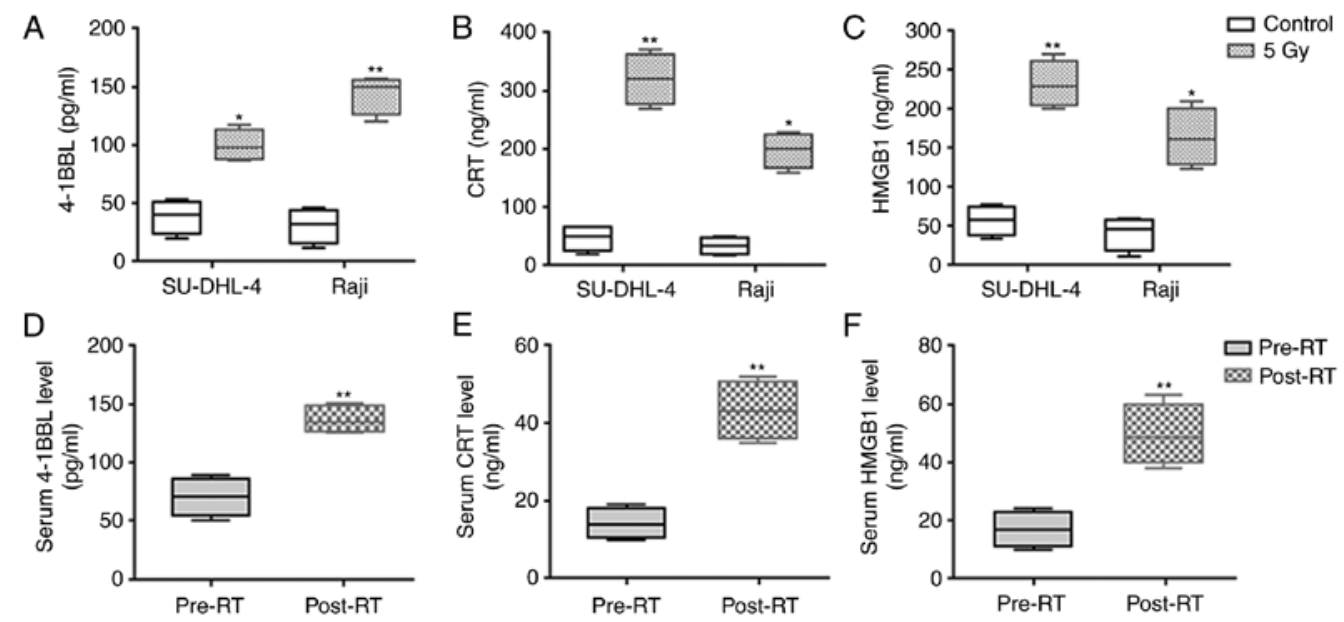

Figure 6. ELISA analysis of protein expressions in cell culture supernatants of non-irradiated or irradiated SU-DHL-4 and Raji cells as well as serum samples of patients with WR-B-NHL prior to and following RT. (A) 4-1BB ligands, (B) CRT and (C) HMGB1 protein in cells. (D) 4-1BB ligands, (E) CRT and (F) HMGB1 protein in serum. ${ }^{*} \mathrm{P}<0.05$ vs. Control; ${ }^{* *} \mathrm{P}<0.01$ vs. Control. ELISA, enzyme-linked immuno sorbent assay; WR-B-NHL, B cell non-Hodgkin's lymphoma of Waldeyer's ring; CRT, calreticulin; HMGB1, high mobility group box 1.
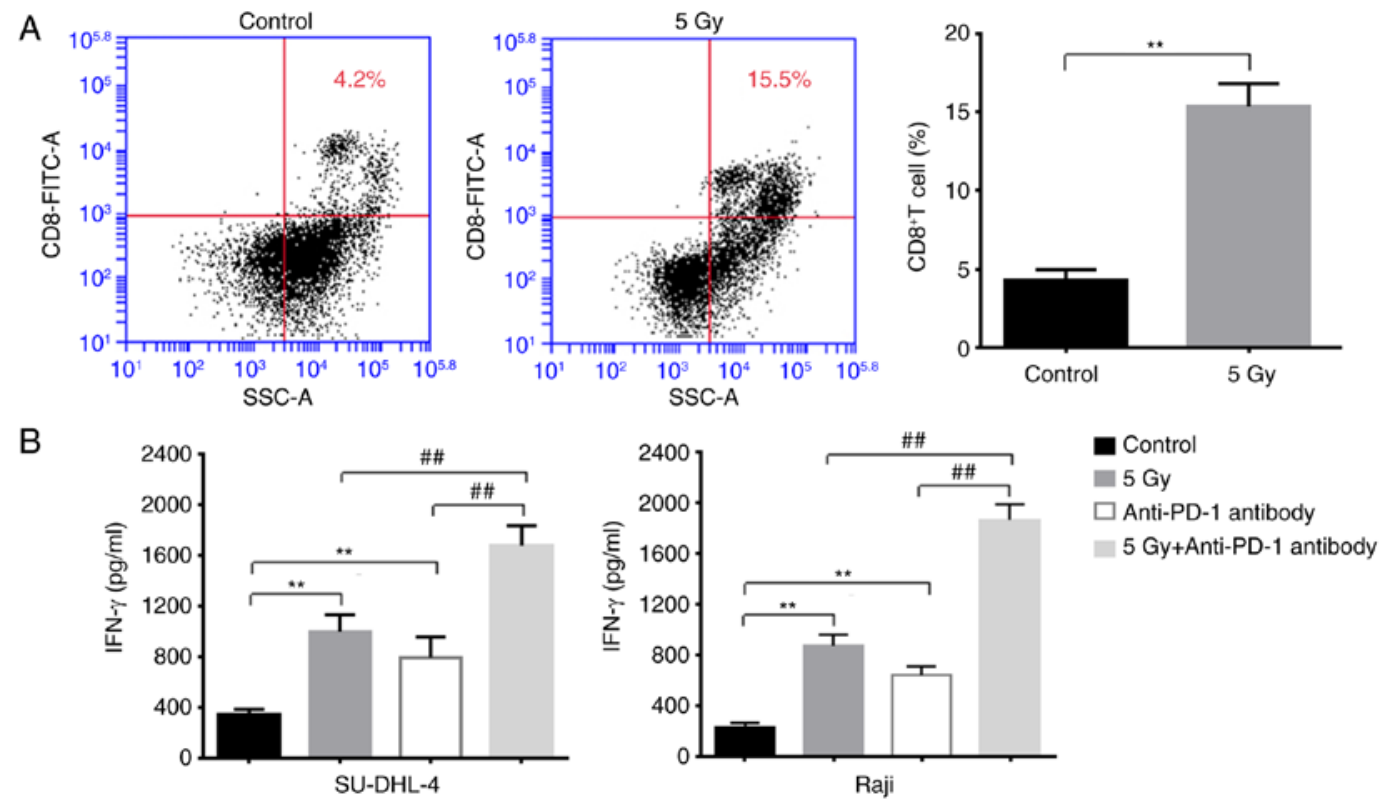

Figure 7. Effects of irradiated SU-DHL-4 and Raji cells on the T cells. (A) Flow cytometry analysis the proportion of CD8 ${ }^{+} \mathrm{T}$ cells in co-culture system of $\mathrm{T}$ cells with non-irradiated or irradiated SU-DHL-4 and Raji cells. (B) ELISA analysis of IFN- $\gamma$ expressions in co-culture system of T cells with SU-DHL-4 and Raji cells treated with non-irradiation, irradiation or the anti-PD-1 antibody. ${ }^{* *} \mathrm{P}<0.01$ vs. control; ${ }^{\# \#} \mathrm{P}<0.01$ vs. 5 Gy and anti-PD-1 antibody.

at mRNA levels, were further detected in the cell supernatants and serum samples of patients with WR-B-NHL. As expected, these proteins were significantly increased in radiated cell supernatants as well as serum samples of RT-treated patients, as demonstrated in Fig. 6.

Reactivity of $T$ effector cells is activated by irradiated lymphoma cells. In order to elucidate the relevance in terms of the radiation lymphoma cells affecting the $\mathrm{T}$ cell anti-tumor responses, $\mathrm{T}$ cells were co-cultured with non-irradiated or irradiated B-lymphoma cells. As presented in Fig. 7A, the proportion of $\mathrm{CD}^{+} \mathrm{T}$ cells was significantly increased when $\mathrm{T}$ cells were co-cultured with irradiated lymphoma cells compared with non-irradiated cells. Furthermore, the expression levels of IFN- $\gamma$ were significantly increased in the irradiation co-culture system (Fig. 7B), suggesting increased anti-tumor activities of T cells. Finally, the anti-PD-1 antibody was added to the co-culture system, which revealed that the expression of IFN- $\gamma$ was significantly elevated in the RT-treated lymphoma-T cell co-culture system, indicating the significant benefits of using anti-PD-1 antibody in RT-treated lymphoma cells (Fig. 7B).

\section{Discussion}

In the present study, a retrospective analysis in a consecutive cohort of 164 patients with WR-B-NHL who underwent RT was performed, which demonstrated that a high LMR is an 
independent favorable systemic immune prognostic factor for clinical RT response and affects the survival outcomes of patients with distant lesions. To the best of our knowledge, this is the first study to estimate the prognostic value of the LMR in RT-treated patients with WR-B-NHL and investigate the potential application of the LMR combined with RT on the systemic immune response, as well as the combination of RT with immunotherapies.

Accumulating studies have demonstrated that survival outcomes in patients with lymphoma are associated with systemic inflammation (22-24,30-32). The peripheral inflammatory cells, including lymphocytes and monocytes, represent significant markers of systematic inflammatory response (20-23). Lymphocytes serve an important role in activating anti-tumor immunity, whereas a decreased number of lymphocytes may result in a poor immunologic response, thus promoting the proliferation and the migration of tumor cells $(33,34)$. Opposite to the lymphocytes, monocytes exert a protumoral effect by infiltrating tumor sites and differentiating into tumor-associated macrophages, which promote tumor growth via secretion of growth factors and immune suppressive cytokines $(35,36)$. Therefore, the LMR may be a better biomarker to evaluate the immune activities of the host. As reported, the LMR may reflect the degree of systemic immunity and is regarded as an important prognostic indicator in a variety of malignancies, including B-NHL (22-24). There are several studies focusing on the role of the LMR on therapeutic responsiveness of chemotherapy regimens $(30,37)$; however, few studies have compared the LMR with clinical response to RT (38), which is also an important therapeutic modality to treat patients with WR-B-NHL. As a result, the purpose of the present study was to investigate whether the LMR predicted the tumor responses and outcomes of patients with WR-B-NHL who received RT.

The optimal cut-off LMR value of 3.14 was selected prior to RT treatment, and it was demonstrated that a high LMR predicted more improved clinical outcomes in patients with WR-B-NHL compared with a low LMR. Furthermore, maintenance of a high LMR was considered a favorable independent prognostic factor for the long-term survival outcomes in RT-treated patients. It is known that RT regulates local tumor burden and elicits anti-tumor immune effects (11). However, there were suggestions that RT alone was not sufficient to initiate anti-tumor effects clinically; thus, the combined treatment of immunotherapy and RT may be more effective $(11,12,39)$. The survival analysis data suggested that a high LMR was a prognostic biomarker for RT.

The present study results also demonstrated the effect of the LMR in RT-treated patients regarding clinical stages and distant lesions. Following the analysis of the prognosis of these subgroups of patients according to the LMR, patients who had a high LMR exhibited a more improved clinical response to RT, which confirmed the prognostic value of the LMR in RT-treated patients with WR-B-NHL. Furthermore, the results of survival analysis of patients with distant lesions indicated that when the patient immune status according to the LMR level is relatively well, RT affected distant non-irradiated fields. This observation may be explained by the 'abscopal effect', whereby localized RT is able to promote systemic immune responses at distant non-irradiated sites $(10,40,41)$.
Although the exact biological mechanisms underlying this effect are not fully elucidated, immune modulation has been reported in several studies $(40,41)$.

In order to explore how RT affects local or systemic immune system, irradiated lymphoma cells were investigated in the current study. The results demonstrated that 4-1BBL, CRT, HMGB1 and OX-40L were significantly overexpressed following irradiation of cells, and 4-1BBL and OX-40L are important co-stimulatory molecules in regulating $\mathrm{T}$ cell function $(29,42)$. Activation of 4-1BBL and OX-40L increases T-cell generation and survival, and enhances T-cell killing of tumor cells (42). CRT and HMGB1 are important hallmarks of immunogenic cell death, which may promote the recruitment of dendritic cells (DCs) into the tumor, the uptake of dying tumor cells by DCs, and efficient antigen presentation to T cells (11). When a tumor is irradiated, radiation-associated antigens are overexpressed and further released. The release of the antigens may infiltrate blood vessels and enter the peripheral blood; thus, they may be able to stimulate the circular T lymphocytes, particularly the $\mathrm{CD}^{+} \mathrm{T}$ cells, the major anti-tumor effector cells. In this case, if the patient has a high LMR, this effect might be enhanced. Thus, the combination of RT with a high LMR may have the capability to prime stronger anti-tumor responses that could control or suppress systemic distant lesion involvements.

At present, RT combined with chemotherapy is still the standard therapy in WR-B-NHL patients. However, as with the development of the immune therapy, the immune checkpoint inhibitors have shown potential application values in lymphoma treatments (43-45). In the present study, whether the anti-PD-1 antibody was able to enhance the anti-tumor effects of T lymphocytes was investigated. Previous studies have shown that the expression of PD-1 is usually induced on activated T cells (46). Therefore, in our co-culture system where $\mathrm{T}$ cells were activated by the irradiated lymphoma cells, the PD-1 expression would increased, and blockade of PD-1 was able to enhance the anti-cancer immune activity of the $\mathrm{T}$ cells, as well as the IFN- $\gamma$ expression (47). Furthermore, we demonstrated better therapeutic effects of combining the anti-PD-1 antibody with RT in the lymphoma cells, which might provide some evidences of using PD-1 antibody in RT-treated lymphomas. In the future, large scale clinical trials are still needed to evaluate the efficacy of combination RT and anti-PD-1 antibody in lymphoma treatments.

In conclusion, the data of the present study demonstrated the predictive significance of the LMR in patients with WR-B-NHL receiving RT and that the LMR may enhance RT sensitivity in patients, particularly those with distant lesions. In this study, the LMR was identified as an independent prognostic biomarker of RT-mediated systemic immune response in treating patients with WR-B-NHL.

\section{Acknowledgements}

Not applicable.

\section{Funding}

The present study was supported by The National Natural Science Foundation of China (grant nos. 81372839 and 81672599), 
and The National Science Foundation of Heilongjiang Province of China for Returness (grant no. LC2017037).

\section{Availability of data and materials}

All data generated or analyzed during this study are included in this published article.

\section{Authors' contributions}

$\mathrm{XN}$ designed and performed the experiments, analyzed the data, and contributed in writing the manuscript; HJ designed and analyzed the data, and contributed in writing; YW, SH, QX, LH, LY, WS, LL, HZ, JL, YY and WA performed the experiments and analyzed the data; QZ designed and supervised the research, analyzed the data, and wrote the manuscript. All authors read and approved the final manuscript.

\section{Ethics approval and consent to participate}

The study protocol was approved by the Institutional Review Board of Harbin Medical University Ethics Committee. Written informed consent was obtained from all participants, whereby informed consent from patients $<16$ years old was obtained from their parents or guardians. All methods were performed in accordance with the relevant guidelines and regulations.

\section{Patient consent for publication}

Not applicable.

\section{Competing interests}

The authors declare that they have no competing interests.

\section{References}

1. Armitage JO, Gascoyne RD, Lunning MA and Cavalli F: Non-Hodgkin lymphoma. Lancet 390: 298-310, 2017.

2. Qi SN, Li YX, Wang H, Wang WH, Jin J, Song YW, Wang SL, Liu YP, Zhou LQ and Yu ZH: Diffuse large B-cell lymphoma: Clinical characterization and prognosis of Waldeyer ring versus lymph node presentation. Cancer 115: 4980-4989, 2009

3. Wu RY, Li YX, Wang WH, Jin J, Wang SL, Liu YP, Song YW, Fang H, Ren H, Liu QF, et al: Clinical disparity and favorable prognoses for patients with Waldeyer ring extranodal nasal-type NK/T-cell lymphoma and diffuse large B-cell lymphoma. Am J Clin Oncol 37: 41-46, 2014

4. Xu YG, Qi SN, Wang SL, Liu YP, Wang WH, Jin J, Song YW, Ren H, Fang H, He XH, et al: Dosimetric and clinical outcomes with intensity modulated radiation therapy after chemotherapy for patients with early-stage diffuse large B-cell lymphoma of Waldeyer ring. Int J Radiat Oncol Biol Phys 96: 379-386, 2016.

5. Phan J, Mazloom A, Medeiros LJ, Zreik TG, Wogan C, Shihadeh F, Rodriguez MA, Fayad L, Fowler N, Reed V, et al: Benefit of consolidative radiation therapy in patients with diffuse large B-cell lymphoma treated with R-CHOP chemotherapy. J Clin Oncol 28: 4170-4176, 2010.

6. Moser EC, Kluin-Nelemans HC, Carde P, Meerwaldt JH, Tirelli U, Aleman BM, Baars J, Thomas J, van Glabbeke $M$ and Noordijk EM: Impact of involved field radiotherapy in partial response after doxorubicin-based chemotherapy for advanced aggressive non-Hodgkin's lymphoma. Int J Radiat Oncol Biol Phys 66: 1168-1177, 2006.

7. Horning SJ, Weller E, Kim K, Earle JD, O'Connell MJ, Habermann TM and Glick JH: Chemotherapy with or without radiotherapy in limited-stage diffuse aggressive non-Hodgkin's lymphoma: Eastern Cooperative Oncology Group study 1484 J Clin Oncol 22: 3032-3038, 2004.
8. Bonnet C, Fillet G, Mounier N, Ganem G, Molina TJ, Thiéblemont C, Fermé C, Quesnel B, Martin C, Gisselbrecht C, et al: CHOP alone compared with CHOP plus radiotherapy for localized aggressive lymphoma in elderly patients: A study by the Groupe d'Etude des Lymphomes de l'Adulte. J Clin Oncol 25: 787-792, 2007.

9. Franceschini D, Franzese C, Navarria P, Ascolese AM, De Rose F, Del Vecchio M, Santoro A and Scorsetti M: Radiotherapy and immunotherapy: Can this combination change the prognosis of patients with melanoma brain metastases? Cancer Treat Rev 50: $1-8,2016$.

10. Yoshimoto Y, Suzuki Y, Mimura K, Ando K, Oike T, Sato H, Okonogi N, Maruyama T, Izawa S, Noda SE, et al: Radiotherapy-induced anti-tumor immunity contributes to the therapeutic efficacy of irradiation and can be augmented by CTLA-4 blockade in a mouse model. PLoS One 9: e92572, 2014.

11. Herrera FG, Bourhis J and Coukos G: Radiotherapy combination opportunities leveraging immunity for the next oncology practice. CA Cancer J Clin 67: 65-85, 2017.

12. Weichselbaum RR, Liang H, Deng L and Fu YX: Radiotherapy and immunotherapy: A beneficial liaison? Nat Rev Clin Oncol 14: 365-379, 2017.

13. Galluzzi L, Kepp O and Kroemer G: Immunogenic cell death in radiation therapy. Oncoimmunology 2: e26536, 2013.

14. Bloy N, Pol J, Manic G, Vitale I, Eggermont A, Galon J, Tartour E, Zitvogel L, Kroemer G and Galluzzi L: Trial Watch: Radioimmunotherapy for oncological indications. Oncoimmunology 3: e954929, 2014.

15. Surace L, Lysenko V, Fontana AO, Cecconi V, Janssen H, Bicvic A, Okoniewski M, Pruschy M, Dummer R, Neefjes J, et al: Complement is a central mediator of radiotherapy-induced tumor-specific immunity and clinical response. Immunity 42: 767-777, 2015.

16. Kroemer G, Galluzzi L, Kepp O and Zitvogel L: Immunogenic cell death in cancer therapy. Annu Rev Immunol 31: 51-72, 2013.

17. Ebner DK, Tinganelli W, Helm A, Bisio A, Yamada S, Kamada T, Shimokawa T and Durante M: The immunoregulatory potential of particle radiation in cancer therapy. Front Immunol 8: 99, 2017.

18. Demaria S, Golden EB and Formenti SC: Role of local radiation therapy in cancer immunotherapy. JAMA Oncol 1: 1325-1332, 2015.

19. Shibutani M, Maeda K, Nagahara H, Iseki Y, Ikeya T and Hirakawa K: Prognostic significance of the preoperative lymphocyte-to-monocyte ratio in patients with colorectal cancer. Oncol Lett 13: 1000-1006, 2017.

20. Ji H, Xuan Q, Yan C, Liu T, Nanding A and Zhang Q: The prognostic and predictive value of the lymphocyte to monocyte ratio in luminal-type breast cancer patients treated with CEF chemotherapy. Oncotarget 7: 34881-34889, 2016.

21. Eo WK, Chang HJ, Kwon SH, Koh SB, Kim YO, Ji YI, Kim HB, Lee JY, Suh DS, Kim KH, et al: The lymphocyte-monocyte ratio predicts patient survival and aggressiveness of ovarian cancer. J Cancer 7: 289-296, 2016.

22. Marconato L, Martini V, Stefanello D, Moretti P, Ferrari R, Comazzi S, Laganga P, Riondato F and Aresu L: Peripheral blood lymphocyte/monocyte ratio as a useful prognostic factor in dogs with diffuse large B-cell lymphoma receiving chemoimmunotherapy. Vet J 206: 226-230, 2015.

23. Wang L, Wang H, Xia ZJ, Huang HQ, Jiang WQ, Lin TY and Lu Y: Peripheral blood lymphocyte to monocyte ratio identifies high-risk adult patients with sporadic Burkitt lymphoma. Ann Hematol 94: 1645-1654, 2015.

24. Ji H, Niu X, Yin L, Wang Y, Huang L, Xuan Q, Li L, Zhang H, Li J, Yang Y, et al: Ratio of immune response to tumor burden predicts survival via regulating functions of lymphocytes and monocytes in diffuse large B-cell lymphoma. Cell Physiol Biochem 45: 951-961, 2018.

25. Campo E, Swerdlow SH, Harris NL, Pileri S, Stein H and Jaffe ES: The 2008 WHO classification of lymphoid neoplasms and beyond: Evolving concepts and practical applications. Blood 117: 5019-5132, 2011.

26. Cassidy RJ, Jegadeesh N, Switchenko J, Danish H, Esiashvili N, Flowers CR and Khan MK: The role of radiotherapy for patients over age 60 with diffuse large B-cell lymphoma in the rituximab era. Leuk Lymphoma 57: 1876-1882, 2016.

27. Lee ST, Liu S, Radvanyi L, Sukhumalchandra P, Molldrem JJ, Wieder ED, Hwu P, Liu YJ, Kwak LW, Lizée G and Neelapu SS: A novel strategy for rapid and efficient isolation of human tumor-specific $\mathrm{CD}^{+}$and $\mathrm{CD}^{+}{ }^{+} \mathrm{T}$-cell clones. J Immunol Methods 331: 13-26, 2008. 
28. Livak KJ and Schmittgen TD: Analysis of relative gene expression data using real-time quantitative PCR and the $2^{-\Delta \Delta C_{\mathrm{T}}}$ method. Methods 25: 402-408, 2001.

29. Sharabi AB, Lim M, DeWeese TL and Drake CG: Radiation and checkpoint blockade immunotherapy: Radiosensitisation and potential mechanisms of synergy. Lancet Oncol 16: e498-e509, 2015.

30. Zhou S, Xu L, Ma Y, Tang L, Zhang Y, Shi Y, Sun L, Chen Y, Liang B, Zhou Y, et al: Peripheral blood lymphocyte to monocyte ratio recovery from low levels at diagnosis after completion of first line therapy predicts good clinical outcomes in patients with diffuse large B-cell lymphoma. Oncotarget 8: 19556-19565, 2017.

31. Ho CL, Lu CS, Chen JH, Chen YG, Huang TC and Wu YY: Neutrophil/Lymphocyte Ratio, Lymphocyte/Monocyte Ratio, and Absolute Lymphocyte Count/Absolute monocyte count prognostic score in diffuse large B-cell lymphoma: Useful prognostic tools in the rituximab era. Medicine 94: e993, 2015.

32. Jia T, Zhang R, Zhu HY, Liang JH, Wang L, Wu W, Cao L, Li JY and $\mathrm{Xu} \mathrm{W}$ : Prognostic significance of peripheral blood absolute monocyte count and lymphocyte to monocyte ratio in anaplastic large cell lymphoma. Cancer Biomark: Jun 12, 2018 (Epub ahead of print).

33. Mantovani A, Allavena P, Sica A and Balkwill F. Cancer-related inflammation. Nature 454: 436-444, 2008.

34. Shibutani M, Maeda K, Nagahara H, Ohtani H, Sakurai K, Yamazoe S, Kimura K, Toyokawa T, Amano R, Tanaka H, et al: Prognostic significance of the lymphocyte-to-monocyte ratio in patients with metastatic colorectal cancer. World J Gastroenterol 21: 9966-9973, 2015.

35. Noy R and Pollard JW. Tumor-associated macrophages: From mechanisms to therapy. Immunity 41: 49-61, 2014.

36. Quail DF and Joyce JA: Microenvironmental regulation of tumor progression and metastasis. Nat Med 19: 1423-1437, 2013.

37. Lin GN, Liu PP, Liu DY, Peng JW, Xiao JJ and Xia ZJ: Prognostic significance of the pre-chemotherapy lymphocyte-to-monocyte ratio in patients with previously untreated metastatic colorectal cancer receiving FOLFOX chemotherapy. Chin J Cancer 35: 5 , 2016.
38. Luo H, Ge H, Cui Y, Zhang J, Fan R, Zheng A, Zheng X and Sun Y: Systemic inflammation biomarkers predict survival in patients of early stage Non-small cell lung cancer treated with stereotactic ablative radiotherapy-a single center experience. J Cancer 9: 182-188, 2018.

39. Dovedi SJ, Lipowska-Bhalla G, Beers SA, Cheadle EJ, Mu L, Glennie MJ, Illidge TM and Honeychurch J: Antitumor efficacy of radiation plus immunotherapy depends upon dendritic cell activation of effector $\mathrm{CD}^{+} \mathrm{T}$ cells. Cancer Immunol Res 4 : 621-630, 2016.

40. Golden EB, Chhabra A, Chachoua A, Adams S, Donach M, Fenton-Kerimian M, Friedman K, Ponzo F, Babb JS, Goldberg J, et al: Local radiotherapy and granulocyte-macrophage colony-stimulating factor to generate abscopal responses in patients with metastatic solid tumours: A proof-of-principle trial. Lancet Oncol 16: 795-803, 2015.

41. Postow MA, Callahan MK, Barker CA, Yamada Y, Yuan J, Kitano S, Mu Z, Rasalan T, Adamow M, Ritter E, et al: Immunologic correlates of the abscopal effect in a patient with melanoma. N Engl J Med 366: 925-931, 2012.

42. Kumari A and Garnett-Benson C: Effector function of CTLs is increased by irradiated colorectal tumor cells that modulate OX-40L and 4-1BBL and is reversed following dual blockade. BMC Res Notes 9: 92, 2016.

43. de Winde CM, Elfrink S and van Spriel AB: Novel insights into membrane targeting of B cell lymphoma. Trends Cancer 3: 442-453, 2017.

44. Jelinek T, Mihalyova J, Kascak M, Duras J and Hajek R: PD-1/PD-L1 inhibitors in haematological malignancies: Update 2017. Immunology 152: 357-371, 2017.

45. Mitteldorf C, Berisha A, Pfaltz MC, Broekaert SMC, Schön MP, Kerl $\mathrm{K}$ and Kempf W: Tumor microenvironment and checkpoint molecules in primary cutaneous diffuse large B-cell lymphoma-new therapeutic targets. Am J Surg Pathol 41: 998-1004, 2017.

46. Boussiotis VA, Chatterjee P and Li L: Biochemical signaling of pd-1 on T cells and its functional implications. Cancer J 20: 265-271, 2014.

47. Nemoto $\mathrm{Y}$, Shosu K, Okuda M, Noguchi S and Mizuno T: Development and characterization of monoclonal antibodies against canine PD-1 and PD-L1. Vet Immunol Immunopathol 198 19-25, 2018. 